\title{
HAART Use and Cardiovascular Risk Among HIV Patients in Rivers State, Nigeria
}

\author{
Ajala Aisha Oluwabunmi, Ofori Sandra Nnedinma, Odia Osarentin James \\ Department of Internal Medicine, University of Port Harcourt Teaching Hospital, Choba, Rivers State, Nigeria
}

Email address:

ajalabunmi@gmail.com (A. A. Oluwabunmi)

\section{To cite this article:}

Ajala Aisha Oluwabunmi, Ofori Sandra Nnedinma, Odia Osarentin James. HAART Use and Cardiovascular Risk Among HIV Patients in Rivers State, Nigeria. Cardiology and Cardiovascular Research. Vol. 3, No. 3, 2019, pp. 45-51. doi: 10.11648/j.ccr.20190303.11

Received: June 26, 2019; Accepted: July 24, 2019; Published: August 7, 2019

\begin{abstract}
Cardiovascular diseases have been associated with deaths among HIV seropositive persons in low income countries. This was a cross-sectional study of cardiovascular risk among 100 HIV seropositive persons and 100 age and sex matched seropositive but HAART-naive controls in Rivers state, Nigeria. The study subjects underwent clinical examinations to determine their blood pressure and anthropometric parameters. Blood samples were taken to assess fasting blood glucose and lipid profile. Risk assessment was done using the WHO/ISH cardiovascular risk score chart. Among the subjects, 76 (76\%) were on a non-PI based HAART compared with $24(24 \%)$ on a PI-based HAART. The mean BMI and CD4 count were significantly higher among the cases than the controls $(\mathrm{p}=.0 .048$ and $\mathrm{p}<0.0001$ respectively). There was a statistically significant difference in the mean SBP $(131.90 \pm 14.33 \mathrm{mmHg}$ versus $127.48 \pm 12.03 \mathrm{mmHg})$ and DBP $(83.88 \pm 6.59 \mathrm{mmHg}$ versus $80.63 \pm 6.74 \mathrm{mmHg})$ between the cases and controls $(\mathrm{p}=0.019$ and 0.023 respectively). Dyslipidaemia was higher among the cases than the controls (low HDL-c was $36 \%$ vs $33 \%$; increased TC $20 \%$ vs $7 \%$; increased LDL-c $13 \%$ vs $4 \%$ and triglycerides $7 \%$ vs $5 \%$. The prevalence of hypercholesterolemia and increased LDL-c was significantly higher among the cases than the controls $(\mathrm{p}=0.007$ and 0.022 respectively). The prevalence of intermediate to high $\mathrm{CV}$ risk score was higher among the HAART experienced HIV seropositive subjects compared to control subjects.
\end{abstract}

Keywords: Cardiovascular Risk, HAART, HIV

\section{Introduction}

Cardiovascular disease (CVD) is a heterogeneous group of diseases that involve the heart and/or blood vessels. Cardiovascular disease includes coronary artery diseases (CAD) such as angina and myocardial infarction, stroke, hypertensive heart disease, rheumatic heart disease, cardiomyopathy, atrial fibrillation, congenital heart disease, endocarditis, aortic aneurysms, peripheral artery disease and venous thrombosis $[1,2]$. Cardiovascular diseases are the leading cause of death globally and will continue to dominate mortality trends in the future [1]. By 2015, the total number of cardiovascular disease (CVD) deaths (mainly coronary heart disease, stroke, and rheumatic heart disease) had increased globally to 17.7 million (31\%) from $12.3(25.8 \%)$ million in 1990. Of these, 7.4 million were attributed to coronary heart disease and 6.7 million to stroke. More than $80 \%$ of the deaths occurred in low-and middle-income countries $[2,3]$.
CVD alone will be responsible for more deaths in low income countries than infectious diseases (including HIV/AIDS, tuberculosis, and malaria), maternal and perinatal conditions, and nutritional disorders combined [4]. Although the CVD burden in Sub-Saharan Africa has risen significantly over the past two decades, available data for most of Africa is scarce due to significant barriers to estimate CVD in this region, including the lack of vital registration systems in most countries [5]. In Nigeria, although CVD lags behind infectious disease as the commonest cause of death, it accounts for higher age-specific mortality rates when compared to developed countries [6].

Patients with HIV infection can have a variety of cardiac and vascular manifestations. However, certain cardiac disorders have been reported with greater frequency among HIV-infected patients compared with the general population [7]. With more effective and widespread treatment of HIV in resource-rich settings, morbidity and mortality from nonAIDS-related events have surpassed those from AIDS-related 
events. In particular, CVD has emerged as an important cause of death in HIV-infected patients relative to the decreasing incidence of opportunistic disease and with the introduction of highly active antiretroviral therapy [HAART] due to the use of drugs that exert metabolic changes $[8,9]$.

Many Antiretroviral Therapies (ARTs) cause hyperlipidaemia or the lipodystrophy syndrome, which is manifested by peripheral fat wasting (lipoatrophy) and visceral fat accumulation (lipohypertrophy). This is a class effect of the Protease Inhibitors (PIs), and also occurs frequently with the nucleoside reverse transcriptase inhibitors (NRTIs). The NRTIs are also associated with hepatic steatosis related to mitochondrial toxicity. Of the NRTIs, Stavudine mainly causes increased triglycerides (TG), but may also increase low density lipoprotein cholesterol (LDLC) and total cholesterol (TC) [10]. Of the non-nucleoside reverse transcriptase inhibitors (NNRTIs), Efavirenz is more likely to cause hyperlipidaemia than Nevirapine [10]. All of the PIs except Atazanavir have been associated with hyperlipidaemia. Protease inhibitors and in particular Ritonavir-boosted PIs cause elevations of LDL, TC and TGs and a decrease in high density lipoprotein cholesterol (HDLC) $[11]$.

Infection with $\mathrm{HIV}$ is independently associated with an increased risk of cardiovascular disease, [7] furthermore the use of HAART has been shown to cause several metabolic derangements which may have potentially damaging effects on the cardiovascular system. Assessment of CV risk in patients taking Highly Active Antiretroviral Therapy (HAART) is therefore warranted [12]. The overall estimated risk will determine the level of prevention to be instituted ranging from primordial to tertiary levels of prevention.

\section{Methods}

\subsection{Study Setting}

The study was carried out at the Anti-Retroviral clinic of the University of Port Harcourt Teaching Hospital, Rivers State, Nigeria. The hospital is a tertiary hospital located in Port Harcourt and serves as a referral medical centre for neighbouring states.

\subsection{Study Sample}

One hundred previously diagnosed HIV seropositive patients who have been on HAART for 12 months and above presenting at the Anti-retroviral clinic of the University of Port-Harcourt Teaching Hospital were recruited into the study after meeting the eligibility criteria as follows;

Individuals aged above 18 years.

Individuals that have confirmed diagnosis of HIV seropositive.

Individuals that are on HAART for $\geq 12$ months.

For each case recruited into the study, a control of same sex and similar age (+/-2 years) was selected recruited into the study after meeting the eligibility criteria as stated below;

Individuals aged above 18 years,
Individuals that have been confirmed HIV sero-positive and HAART naïve.

Individuals with no pre-existing illnesses.

\subsection{Data Collection and Measurements}

A structured interviewer-administered questionnaire to collect demographic information and disease related variables from the subjects. A detailed physical examination was conducted to determine weight, height, abdominal circumference and blood pressure. Venous blood was taken for fasting lipid profile, fasting blood glucose and serum creatinine from all study subjects. Patients with eGFR $\leq 60 \mathrm{ml} / \mathrm{min}$ and fasting plasma glucose level of $7.0 \mathrm{mmol} / 1$ and above were excluded from participating in the study. Cardiovascular risk score was calculated using the WHO/ISH Risk Prediction Chart for Africa, sub-region D with parameters such as age, sex, systolic blood pressure, history of diabetes, smoking and total cholesterol values, which were used to calculate the 10 -year risk of future cardiovascular events. Scores $<10 \%$ signify low risk, $10-20 \%$ intermediate risk and $>20 \%$ high risk [13]. Impaired fasting glucose (FBG) was defined as FBG between 5.6-6.9mmol/1. LDL cholesterol values were calculated using the Friedewald equation when the triglyceride level was less than $4.0 \mathrm{mmol} / 1: \mathrm{LDL}=\mathrm{TC}-$ (HDL + TG /2.2) [14]. Abnormal lipid profile was defined as elevated triglyceride with $\mathrm{TG}>1.7 \mathrm{mmol} / \mathrm{l}$, hypercholesterolemia with TC $>5.2 \mathrm{mmol} / 1$, low highdensity lipoprotein cholesterol with HDL-c $<1.03 \mathrm{mmol} / 1$ and elevated low-density lipoprotein cholesterol LDL-c $>$ $3.0 \mathrm{mmol} / 1$ [15]. Blood samples were analyzed in the chemical pathology laboratory of UPTH, fasting cholesterol and triglyceride levels were measured using the enzymatic method with a reagent from Atlas Medical Laboratories. Fasting HDL was measured with the precipitation method.

\subsection{Statistical Analysis}

All data were analysed using the Statistical Package for Social Sciences (SPSS) version 20 analytic software. The main outcomes were WHO/ISH CV risk score, while the predictor variable was HAART use (HAART experienced and HAART naïve HIV seropositive patients). Continuous variables such as WC, WHR, BP, TC, TG, HDL-c, calculated LDL-c, FBS, CD4 cell count were analysed using independent sample t-test and results expressed as mean \pm standard deviation, while categorical variables such as BMI category and WHO/ISH CV risk score category were analysed using chi-square test or two tailed Fisher's exact and results expressed as percentages. All tests were considered to be statistically significant at the p-value less than 0.05 .

\subsection{Ethical Consideration}

Ethical approval was obtained from the Ethics and Research Committee of the University of Port Harcourt 
Teaching Hospital (UPTH) prior to commencement of the study. Written informed consent was also obtained from willing participants before they were included in the study. There was no denial of standard clinical care to individuals that refused consent to participate in the study.

\section{Results}

There was no significant difference in the mean age of the cases and the controls $37.28 \pm 8.92$ (range $18-57$ years) versus $36.20 \pm 8.92$ years (range $19-58$ years). The most common age groups among both the cases and controls were the 20-29 years and 30-39 years as shown in Table 1.

Table 1. Socio-demographic characteristics of study subjects.

\begin{tabular}{|c|c|c|c|c|}
\hline Variable & Case $n=100(\%)$ & Control $n=100(\%)$ & Total $n=200(\%)$ & Chi-square (p-value) \\
\hline \multicolumn{5}{|l|}{ SEX } \\
\hline Female & $65(65.0)$ & $61(61.0)$ & $74(37.0)$ & \multirow{2}{*}{$0.558(0.343)$} \\
\hline Male & $35(35.0)$ & $39(39.0)$ & $126(63.0)$ & \\
\hline \multicolumn{5}{|l|}{ AGE GROUP } \\
\hline$<20$ years & $1(1.0)$ & $2(2.0)$ & $3(1.5)$ & \multirow{4}{*}{$6.993(0.136)$} \\
\hline $20-29$ years & $25(25.0)$ & $22(22.0)$ & $47(23.5)$ & \\
\hline $30-39$ years & $41(47.0)$ & $47(41.0)$ & $88(44.0)$ & \\
\hline$\geq 50$ years & $10(10.0)$ & $9(9.0)$ & $19(9.5)$ & \\
\hline \multicolumn{5}{|c|}{ EDUCATIONAL } \\
\hline \multicolumn{5}{|l|}{ STATUS } \\
\hline None & $0(0.0)$ & $1(1.0)$ & $1(0.5)$ & \multirow{4}{*}{$* *(0.745)$} \\
\hline Primary & $20(20.0)$ & $18(18.0)$ & $38(19.0)$ & \\
\hline Secondary & $47(47.0)$ & $52(52.0)$ & $99(49.5)$ & \\
\hline Tertiary & $33(33.0)$ & $29(29.0)$ & $62(31.0)$ & \\
\hline Single & $34(34.0)$ & $45(45.0)$ & $79(39.5)$ & \multirow{4}{*}{$* *(0.354)$} \\
\hline Married & $61(61.0)$ & $49(49.0)$ & $110(55.0)$ & \\
\hline Separated & $1(1.0)$ & $1(1.0)$ & $2(1.0)$ & \\
\hline Divorce & $4(4.0)$ & $5(5.0)$ & $9(4.5)$ & \\
\hline \multicolumn{5}{|l|}{ Use of tobacco } \\
\hline Yes & $4(4.0)$ & $5(5.0)$ & $9(4.5)$ & \multirow{2}{*}{$* *(1.000)$} \\
\hline No & $96(96.0)$ & $95(95.0)$ & $191(95.5)$ & \\
\hline \multicolumn{5}{|l|}{ Use of alcohol } \\
\hline Yes & $21(21.0)$ & $34(34.0)$ & $55(27.5)$ & \multirow{2}{*}{$4.238(0.040)^{*}$} \\
\hline No & $79(79.0)$ & $66(66.0)$ & $145(72.5)$ & \\
\hline
\end{tabular}

*statistically significant, ** Fishers exact.

Figure 1 shows the distribution of the HARRT regimen among the subjects, the number of cases on a non-PI based HAART was $76(76 \%)$, while $24(24 \%)$ were on PI-based HAART.

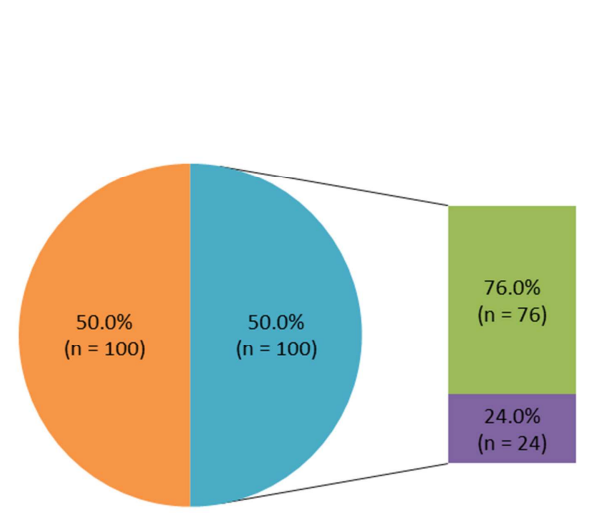

- HAART Nä̈ve - HAART Experienced Non-PI based HAART - PI based HAART

Figure 1. Type of HAART among Study subjects.

The body mass index (BMI) was significantly higher in the cases compared to the controls, $23.39 \pm 2.23 \mathrm{~kg} / \mathrm{m} 2$ versus $21.92 \pm 5.55 \mathrm{~kg} / \mathrm{m} 2(\mathrm{p}=0.048)$. Twenty-three $(23 \%)$ of the cases were classified as overweight compared to $19 \%$ of the controls and $5 \%$ of the cases vs $2 \%$ of the controls had class
1 obesity as shown in Figure 2. The overall prevalence of overweight and obesity in this study was $24.5 \%$.

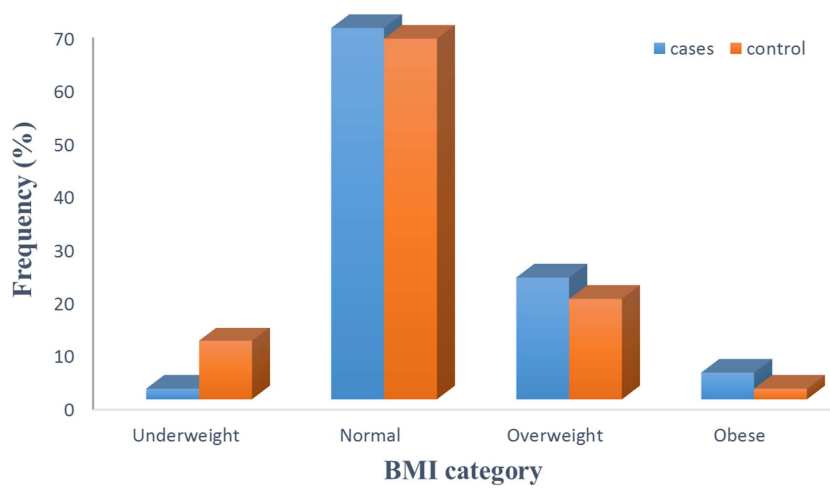

Figure 2. Distribution of BMI Classification in study subjects.

The anthropometric measurement and lipid profile of the study subjects are presented in Table 2 . The differences in the BMI, waist circumference, systolic blood pressure, diastolic blood pressure, total cholesterol, low density lipoprotein and CD4 counts between the HAART experienced and HAART-naïve subjects was found to be statistically significant $(\mathrm{p}<0.05)$. The prevalence of 
overweight, obesity, dyslipidaemia, impaired fasting glucose and high blood pressure were higher in the HAART experienced cases when compared to the controls showed that the distribution of abdominal obesity, hypercholesterolaemia and high low density lipoprotein (LDL-c) was found to be statistically different between the cases and controls as shown in Table 3.

Table 2. Comparison of clinical and laboratory findings among cases and controls.

\begin{tabular}{llll}
\hline Variables & HAART experienced $(\mathbf{n}=\mathbf{1 0 0})$ & HAART Naïve $(\mathbf{n}=\mathbf{1 0 0})$ & Student t-test $($ P value $)$ \\
\hline Age & $37.28 \pm 8.918$ & $36.20 \pm 8.923$ & $0.896(0.393)$ \\
BMI $(\mathrm{kg} / \mathrm{m} 2)$ & $23.39 \pm 2.23$ & $21.92 \pm 5.55$ & $7.926(0.048)^{*}$ \\
WC $(\mathrm{cm})$ & $88.22 \pm 9.79$ & $85.98 \pm 9.57$ & $6.486(0.011)^{*}$ \\
WHR & $0.884 \pm 0.089$ & $0.872 \pm 0.11$ & $0.851(0.396)$ \\
SBP $(\mathrm{mmhg})$ & $131.910 \pm 14.33$ & $127.48 \pm 12.03$ & $2.368(0.019)^{*}$ \\
DBP $(\mathrm{mmhg})$ & $83.88 \pm 6.59$ & $80.63 \pm 6.74$ & $2.289(0.023)^{*}$ \\
FBS $(\mathrm{mmol} / \mathrm{l})$ & $5.28 \pm 0.748$ & $5.21 \pm 0.712$ & $0.764(0.446)$ \\
TC $(\mathrm{mmol} / \mathrm{l})$ & $3.78 \pm 1.193$ & $3.41 \pm 0.941$ & $2.348(0.020)^{*}$ \\
TG $(\mathrm{mmol} / \mathrm{l})$ & $1.064 \pm 0.37$ & $1.028 \pm 0.33$ & $0.736(0.463)$ \\
HDL-c $(\mathrm{mmol} / \mathrm{l})$ & $1.12 \pm 0.39$ & $1.14 \pm 0.38$ & $0.492(0.623)$ \\
LDL-c $(\mathrm{mmol} / \mathrm{l})$ & $1.86 \pm 0.84$ & $1.63 \pm 0.59$ & $2.016(0.045)^{*}$ \\
CD4 count $\left(\mathrm{mm}{ }^{3}\right)$ & $450.31 \pm 260.33$ & $312.55 \pm 207.08$ & $3.751(0.0001)^{*}$ \\
\hline
\end{tabular}

*Statistically significant. Values are mean \pm standard deviation. Key: BMI $=$ body mass index, $\mathrm{WC}=$ waist circumference, $\mathrm{WHR}=$ waist to hip ratio, $\mathrm{SBP}=$ systolic blood pressure, $\mathrm{DBP}=$ diastolic blood pressure, $\mathrm{FBS}=$ fasting blood sugar, $\mathrm{TC}=$ total cholesterol, $\mathrm{TG}=$ triglyceride, $\mathrm{HDL}=$ high density lipoprotein cholesterol, LDL $=$ low density lipoprotein cholesterol.

Table 3. Prevalence of cardiovascular risk factors among cases and controls.

\begin{tabular}{lllll}
\hline Clinical and laboratory findings & Cases $\mathbf{n}=\mathbf{1 0 0}(\mathbf{\%})$ & Controls $\mathbf{n}=\mathbf{1 0 0}(\mathbf{\%})$ & Total $\mathbf{n}=\mathbf{2 0 0}(\mathbf{\%})$ & Chi-square $(\mathbf{p}$ value) \\
\hline Overweight/obese & $28(28.0)$ & $21(21.0)$ & $49(24.5)$ & $1.325(0.250)$ \\
Abdominal obesity & $42(42.0)$ & $25(25.0)$ & $67(33.5)$ & $6.486(0.011)^{*}$ \\
Hypercholesterolaemia & $20(20.0)$ & $7(7.0)$ & $27(13.5)$ & $7.235(0.007)^{*}$ \\
High LDL-c & $13(13.0)$ & $4(4.0)$ & $17(8.5)$ & $5.207(0.022)^{*}$ \\
Low HDL-c & $36(36.0)$ & $33(33.0)$ & $69(34.5)$ & $0.199(0.655)$ \\
Hypertriglyceridemia & $7(7.0)$ & $5(5.0)$ & $12(6.0)$ & $0.355(0.552)$ \\
Hyperglycaemia & $9(9.0)$ & $4(4.0)$ & $13(6.5)$ & $2.057(0.152)$ \\
Hypertension & $18(18.0)$ & $9(9.0)$ & $27(13.5)$ & $3.463(0.063)$ \\
\hline
\end{tabular}

The World Health Organization/International Society for Hypertension CV risk score was low in majority of the respondents. Twelve (12\%) of the cases had an intermediate to high cardiovascular risk score compared with five $(5 \%)$ of the control subjects as illustrated in figure 3 .

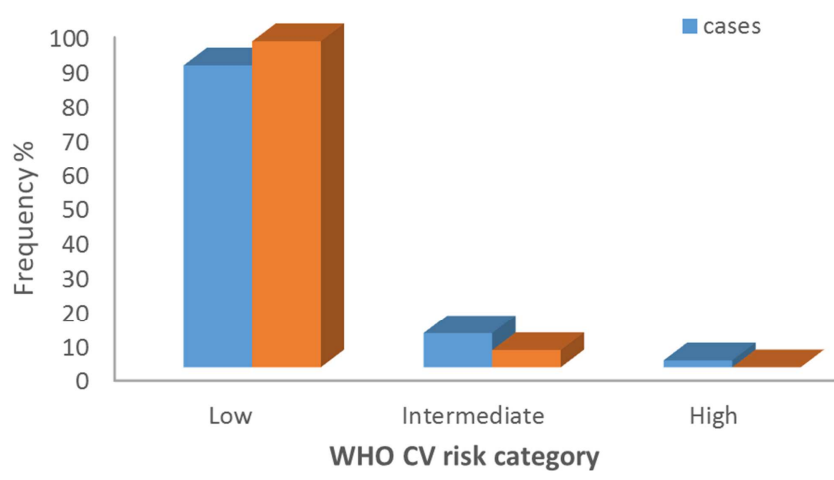

Figure 3. Distribution of cardiovascular risk score among subjects.

\section{Discussion}

Most patients were in the age group 20-29 years and 30-39 years. This was also similar to previous studies done is some tertiary institutions where Edward et al [16] and Osegbe et al [17] found a similar mean age in their study population. The mean age of most adults living with HIV/AIDS is between 15-49 years of age. [16] The HAART experienced cases where mostly on a non-PI based HAART group. Two nucleoside reverse transcriptase inhibitors (NRTI) and one non-nucleoside reverse transcriptase inhibitors are recommended as first line therapy in the treatment of PLWHIV/AIDS and patients are usually switched or substituted to second line therapy due to treatment failure [17]. Osegbe et al in a tertiary institution based study in Lagos, Nigeria also found that 15 out of 100 treated patients were on a PI based HAART regime [17]. Reinsch et al also found that more patients were on a NRTIs based regime than a PI-based regime in a large multicentre study done in Germany [18].

Most of the traditional cardiovascular risk factors (CVRFs) present in the normal population are also present in HIV infected patients, however patients with the conventional risk factors like known hypertension and overt diabetes mellitus were excluded from this study.

The prevalence of overweight and generalized obesity in this study is similar to that reported by Edward et al [16] at $24.2 \%$ and $7.5 \%$ respectively. However, Nsagha et al reported a higher prevalence of $40 \%$ vs $8 \%$ among HAART treated and HAART naïve patients in a study done in Cameroon to assess risk factors for CVD in HIV patients on HAART [1]. Mashinya et al also reported a higher prevalence of obesity at $12.7 \%$ [19].

This high prevalence in the HAART treated group was attributed to HIV patients over-feeding on rich foodstuff so 
as to maintain their weight and immunity status. HIVassociated lipodystrophy syndrome which occurs shortly after introduction of anti-retroviral therapy and includes central adiposity, subcutaneous lipoatrophy, dyslipidaemia and insulin resistance could also explain the increased prevalence of overweight and obesity among HAART experienced patients compared to their HAART naïve counterparts [20]. Many published reports have drawn attention to the increasing prevalence of overweight and obesity in PLWHIV/AIDS due to the fact that being overweight removes the suspicion of HIV positivity and stigma [21].

Many antiretrovirals have dyslipidaemic properties, the use of NRTIs and PIs have been associated with dyslipidaemia in several reported studies [22, 23]. An increased prevalence of hypercholesterolaemia, Low HDL-c and increased LDL-c was found among HAART treated patients in this study, but the prevalence of hypertriglyceridaemia was generally low. The prevalence of hypercholesterolaemia of $20 \%$ in the treatment arm vs $7 \%$ in the naïve group was much lower than values reported by Nsagha et al where hypercholesterolaemia was reported in $40 \%$ of the study population [21]. The higher prevalence by Nsagha et al [21] could be attributed to the higher mean age of the study subjects recruited and also more previously known hypertensive patients were recruited. Nery et al in a Brazillian cross-sectional study reported a low prevalence of isolated hypercholesterolaemia. However, there are suggestions that the magnitude of HIV infection and its treatment induced lipid derangements could vary across populations and settings [24].

The most common dyslipidaemia in this study was low HDL-c found in more than $36 \%$ of the cases versus $33 \%$ of the control subjects. This finding was similar to the study by Mashinya et al in a rural South-African cross-sectional study where a slightly higher prevalence of $43.8 \%$ was reported among the HAART treated patients [22]. Edward et al also reported a similar prevalence of low HDL-c among HAART treated patients [16]. The Data on the Adverse effect on AntiHIV Drugs (DAD) study, a large multicenter prospective study done in 21 countries in Europe, Australia and United States found a slightly lower prevalence of HDL-c at $26.1 \%$. HIV seroconversion has been linked to a reduction in total cholesterol, LDL-c and HDL-c, however initiation of HAART causes a resultant increase in total cholesterol, LDLc and triglyceride but HDL-c has been found to remain low [25]. Some Antiretroviral therapy such as NNRTIs has been shown to result in an increase in HDL-c of approximately $40 \%$.

A high LDL-c was reported in $13 \%$ of the cases versus $4 \%$ of the control group. Nsagha and colleagues in Cameroon however reported a much higher prevalence at 29.3\% [21]. The findings in this study was however comparable but higher than that reported by Nery et al in a Brazilian study. The prevalence of hypertriglyceridaemia was low among the cases and control subjects at $7 \%$ vs $5 \%$. The DAD study reported a high prevalence of $32.2 \%$ [28] Several other reported studies have found a much higher prevalence [21, 27] Edward and colleagues in a teaching hospital based study in South-western, Nigeria also found a low prevalence of elevated triglycerides (TGs). Triglycerides concentrations and TG clearance time in untreated HIV patients have been shown to correlate with increased serum levels of interferonalpha (IFN-alpha) and also, HIV infection causes a downregulation of the activity of lipoprotein lipase (LPL) and hepatic lipase and hence accumulation of TGs in the circulation [26].

The NRTIs and NNRTIs have not been shown to have much effect on TG clearance as much as the PIs. 83 Most patients in this study on HAART were on a combination of 2 NRTIs and an NNRTI. Blacks and people of African descent also tend to have low levels of elevated TGs and this has been ascribed to higher levels of LPL activity uninhibited by insulin resistance and low apolipoprotein C III levels in this group of people compared to the Caucasians. This could likely explain the low prevalence of hypertriglyceridaemia in this study.

The prevalence of hypertension in this study was $18 \%$ vs $9 \%$ among the cases and controls with an overall prevalence of $13.5 \%$. This value falls within the reported prevalence of $11.3 \%-26.2 \%$ in several other local and international studies $[21,22,24]$. The mean SBP and DBP among HAART treated patients were significantly higher than their naïve counterparts. Nsagha et al found the prevalence of hypertension to be two times higher among the HAART treated patients than their HAART naïve counterpart and this was similar to findings in this study [21]. Several other studies have reported similar findings $[27,28]$. The finding of a significant difference in the prevalence of hypertension among the HAART treated and naïve patients could be explained by the increased prevalence of overweight and obesity among the treated patients. It has also been postulated that untreated HIV disease tend to lower BP with a resultant increase in blood pressure on suppression of HIV replication and normalization of immune status [29].

The impact of weight loss on blood pressure is much studied and this appears to be evident among the HAART naïve patients as the mean systolic and diastolic BP were lower among the controls. Although the etiology of this resultant decrease in BP is largely unknown, the tendency to have a lower blood pressure among HIV-HAART naïve patients may however not lower their overall cardiovascular risk.

Prevalence of hyperglycaemia was low in this study as patients with diabetic range FBG were excluded from this study. Nine percent of the cases vs $5 \%$ of the control group had impaired fasting glucose. Several studies have reported a low prevalence of diabetes among their study cohorts ranging from $0-3.0 \%$ despite antiretroviral therapy being linked to insulin resistance [22, 24, 27]. The use of tobacco among cohorts was low at $4 \%$ among the cases vs $5 \%$ in the control and this values were comparable to some studies done in subSaharan Africa [16, 21] Higher smoking prevalence of 
between $21.1 \%$ - $23.1 \%$ was however reported in some other local and foreign studies [22, 27].

The World Health Organization/International Society for Hypertension (WHO/ISH) cardiovascular risk score was low in majority of the study cohorts. The prevalence of intermediate to high $\mathrm{CV}$ risk score of $12 \%$ in the treated arm versus $5 \%$ in the naïve group is comparable to $12.8 \%$ reported by Edward and colleagues. There is limited data on the use of WHO/ISH CV risk score for assessment of CV risk in PLWHIV/AIDS, however the use of this risk score assessment in the general population in as study done by Mendis et al found a low prevalence of $0.2-4.8 \%$ considered as high risk [28]. Several studies have used other risk assessment tool like the FRS, SCORE, ASCVD risk score and the DAD risk equation $[17,22,27]$.

The overall prevalence of cardiovascular risk using this other risk prediction tools has been mostly low and similar to that reported in this study with the exception of the Data collection on Adverse Effect of Anti-HIV Drugs [DAD] risk equation. Nery et al also reported a low prevalence of intermediate to high risk of $6 \%$ using the FRS compared with $25.8 \%$ when the DAD risk equation was used [27]. In the DAD study, a prevalence of $9.9 \%$ was reported using the FRS [28]. Mashinya et al also compared the risk prediction tools in a tertiary institution based South African study and found a much higher prevalence of $31.1 \%$ using the DAD risk equation compared to $6.7 \%$ when the FRS was used [22]. Other risk prediction tools that have been used in assessing $\mathrm{CV}$ risk in PLWHIV/AIDS have mostly reported a low prevalence similar to that reported in this study and these can be explained by the fact that this tools were designed for the general population and not a specific group of patients and doesn't recognize the additional CV risk bestowed on patients from the use of HAART. Most studies have estimated CVD in HIV patients using the FRS and less frequently other risk scores and these tools have been found to either accurately predict, overestimate but mostly underestimate cardiovascular risk.

\section{Conclusion}

This study although limited by the small sample size, has clearly shown that people living with HIV on HAART have a higher prevalence of cardiovascular risk factors (hypertension, dyslipidaemia, overweight/obesity) when compared to age and sex-matched controls who are HIV positive but HAART naïve. These risk factors contribute in a multiplicative manner to increase their overall risk for CVD. The use of the WHO/ISH risk calculator to estimate risk in this population likely underestimates the cardiovascular risk as it does not consider any HIVspecific risk factors. Our study findings are important especially in low-resource settings to sensitise health care providers to assess cardiovascular risk in a consistent manner when administering care to HIV positive patients especially after they are commenced on HAART. Further studies are required to assess the prognostic impact of estimated risk on cardiovascular outcomes in this patient population.

\section{References}

[1] Mendis S, Puska P, Norrving B. Global Atlas on Cardiovascular Disease Prevention and Control, WHO in collaboration with the World Heart Federation and the World Stroke Organization. 2011; 3-18. ISBN 978-92-4-156437-3.

[2] Global 2013 Mortality and all cause of death collaborators. Global, regional, and national age-sex specific all-cause and cause-specific mortality for 240 causes of death, 1990-2013: a systematic analysis for the Global Burden of Disease Study 2013. Lancet 2015; 385 (9963): 117-171.

[3] World Health Organization. Cardiovascular diseases fact sheet, updated May $2017 . \quad$ Available at: http://www.who.int/mediacentre/factsheets/fs317/en/. Assessed May 2017.

[4] Beaglehole R, Bonita R. Global public health: A scorecard. Lancet. 2008; 372 (9654): 1988-1996.

[5] Mensah GA, Roth GA, Sampson UK, Moran AE, Feigin VL, Forouzanfar MH et al. Mortality from cardiovascular diseases in sub-Saharan Africa, 1990-2013: a systematic analysis of data from the Global Burden of Disease Study 2013. Cardiovasc J Afr. 2015; 26: S6-S10.

[6] Yusuf S, Reddy S, Ôunpuu S, Anand S. Global burden of cardiovascular diseases. Part I: General considerations, the epidemiologic transition, risk factors, and impact of urbanization. Circulation. 2001; 104 (22): 2746-2753.

[7] Reinsch N, Neuhaus K, Esser S, Potthoff A, Hower M, Mostardt S et al. Cardiovascular risk factors in HIV: results of the HIV-HEART study. European Journal of Preventive Cardiology. 2012; 19 (2) 267-274.

[8] Neuhaus J, Angus B, Kowalska JD, La Rosa A, Sampson J, Wentworth D et al. INSIGHT SMART and ESPRIT study groups. Risk of all-cause mortality associated with nonfatal AIDS and serious non-AIDS events among adults infected with HIV/AIDS. AIDS 2010; 24 (5): 697-706.

[9] Antiretroviral Therapy Cohort Collaboration, causes of death in HIV-1-infected patients treated with antiretroviral therapy, 1996-2006: collaborative analysis of 13 HIV cohort studies. Clinical Infectious Diseases. 2010; 50 (10): 1387 - 1396.

[10] White AJ. Mitochondrial toxicity and HIV therapy. Sex Transm Infect. 2001; 77 (3): 158-173.

[11] Dube MP, Stein JH, Aberg JA, Fichtenbaum CJ, Gerber JG, Tashima KT et al. Guidelines for the evaluation and management of dyslipidemia in human immunodeficiency virus (HIV)-infected adults receiving antiretroviral therapy: recommendations of the HIV Medical Association of the Infectious Disease Society of America and the Adult AIDS Clinical Trials Group. Clin Infect Dis. 2003; 1 (37 (5): 613627.

[12] Reyskens PM, Essop MF. HIV protease inhibitors and onset of cardiovascular diseases: a central role for oxidative stress and dysregulation of the ubiquitin-proteasome system. Biochims Biophys Acta 2014; 1842 (2): 256-268. 
[13] Mendis S, Lindholm LH, Mancia G, Whitworth J, Alderman M, Lim S et al. World Health Organization and International Society of Hypertension risk prediction charts: Assessment of cardiovascular risk for prevention and control of cardiovascular disease in low and middle-income countries. Journal of Hypertension. 2007; 25: 1578-1582.

[14] Martin S, Blaha M, Elshazly M. Brinton E, Toth P, McEvoy J et al. Friedewald-estimated versus directly measured lowdensity lipoprotein cholesterol and treatment implications Journal of the American College of Cardiology. 2013; 62 (8): 732-739.

[15] Third Report of the National Cholesterol Education Program Expert Panel on Detection, Evaluation and Treatment of High Blood Cholesterol in Adults (Adult Treatment Panel III) final report. Circulation. 2002; 106 (25): 3143-3421.

[16] World Health Organization, Global HIV/AIDS Statistics November 2014. Available at: https://www.aids.gov/hiv-aidsbasics/hiv-aids-101/global-statistics. Assessed on June 2017.

[17] Rathbun CR, Liedtke MD, Staci ML. Antiretroviral Therapy in HIV infection. Medscape. Available at: http://emedicine.medscape.com/article/1533218. Accessed on January, 2019.

[18] Nsagha DS, Assob JC, Njunda LA, Tanue EA, Kibu DO, Ayima WC et al. Risk factors of cardiovascular diseases in HIV/AIDS patients on HAART. AIDS. 2015; 9: 51-59.

[19] Mashinya F, Alberts M, Colebunders R. Assessment of cardiovascular risk factors in people with HIV infection treated with ART in rural South Africa: a cross sectional study. AIDS Research and Therapy. 2015; 12 (1): 42-52.

[20] Feeney RE, Mallon PW. HIV and HAART - Associated Dyslipidema. The Open Cardiovascular Medicine Journal. 2011; 5: 49-63.

[21] Muhammad S, Sani MU, Okeahialam BN. Cardiovascular disease risk factors among HIV-infected Nigerians receiving Highly Active Antiretroviral Therapy. Nigerian Medical Journal. 2013; 54 (3): 185-190.
[22] Danwe C, Atchou G, Nkam M, Mbuagbaw J, Mougnoutou R, Nkouanfack C et al. Effect of Antiretroviral Therapy on Lipid Metabolism in HIV/AIDS Subjects in Cameroon. Journal of Medical Sciences. 2005; 5 (2): 78-82.

[23] Riddler SA, Smit E, Cole SR, Li R, Chmiel JS, Dobs A et al. Impact of HIV infection and HAART on serum lipids in men. JAMA. 2003; 289 (22): 2978-2982.

[24] Nery MW, Martelli TM, Silveira EA, Oliveira Falco M, Oliveira de Castro CA, Esper JT et al. Cardiovascular Risk Assessment: A Comparison of the Framingham, PROCAM, and DAD Equations in HIV-Infected Persons," The Scientific World Journal. 2013: Article ID 969281.

[25] Friis-Møller N, Sabin CA, Weber R, D'Arminio Monforte A, El-Sadr WM, Reiss P et al. Combination antiretroviral therapy and the risk of myocardial infarction. Data Collection on Adverse Events of Anti-HIV Drugs (DAD) Study Group. N Engl J Med. 2003; 349 (21): 93-103.

[26] Dube MP, Stein JH, Aberg JA, Fichtenbaum CJ, Gerber JG, Tashima KT et al. Guidelines for the evaluation and management of dyslipidemia in human immunodeficiency virus (HIV)-infected adults receiving antiretroviral therapy: recommendations of the HIV Medical Association of the Infectious Disease Society of America and the Adult AIDS Clinical Trials Group. Clin Infect Dis. 2003; 1 (37 (5)): 613627.

[27] Obirikorang C, Quaye L, Osei-Yeboah J, Odame EA, Asare I. Prevalence of metabolic syndrome among HIV-infected patients in Ghana: A cross-sectional study. Niger Med J. 2016; 57 (2): 86-90.

[28] Ghorpade AG, Shrivastava SR, Kar SS, Sarkar S, Majgi SM, Roy G. Estimation of the cardiovascular risk using World Health Organization/International Society of Hypertension (WHO/ISH) risk prediction charts in a rural population of South India. Int J Health Policy Manag. 2015; 4 (8): 531-536. 